\section{The Pollyanna report}

\section{F.J. Bollum}

Soviet Progress in Biochemistry. Soviet editor-in chief V.K. Lishko. Allerton, New York. 6/yr. \$395.

THE scope of this translation series is less than would be expected from the title, because it includes only selected articles from the Ukrainian Biochemical Journal rather than providing a comprehensive view of biochemistry in the Soviet Union*. As such it is an adjunct to the translation of Biokhemika, with which it shares some technical problems. Thus my most serious criticism is of the American publishers for producing this journal of substandard translation by substandard printing methods. Photo-offset printing of typed manuscripts and poor scaling and reproduction of figures makes for hard, sometimes impossible, reading. And the quality of translation requires additional concentration. One expects much better, given the state of computer-assisted wordprocessing and translation.

The papers translated include original *From 1989, Soviet Progress in Biochemistry will be retitled $U$ krainian Biochemistry.

\section{Metastic Spread of Cancer \\ G.V. Sherbet}

This book outlines a new approach to the analysis of the mechanisms of metastasis by comparing the glioma, an intracranial tumour which ordinarily does not metastasize, to other metastasizing tumour models. This has produced a coherent picture of the operation of oncogenes and growth factors in the initiation, development and progression of neoplasms. This is an essential book for those researching metastasis and will be of interest to all research oncologists.

\section{$1987 \quad \mathbf{f} 45.00 \quad 275 p p$ \\ $234 \times 156 \mathrm{~mm} \quad 0333443624$}

work, brief communications and reviews of new methodology. Although these are said to be 'selected articles', there is no indication of the selection process, nor that anything less than the complete journal has been translated in the four issues I reviewed. The issues were published in 1987 , so the experimental work reported in them was probably completed in 1985 1986. This double publication lag is expected in translation, but does put some bias on the reader's view of the currency of the information.

Forgetting for the moment all of these technical problems, what is going on in Ukrainian biochemistry? By Western European and American standards - not too much. One review article describes the virtues of immunoblot technology. With the extended uses of solid-phase substrates current in Western biochemistry (no pun intended), this article provides no new insight. The original papers often have an applied tone, being "The effect of [compound A] on the metabolism of [substance B]". One interesting example providing little comfort to space travellers and embassy employees is
"The Effect of Alternating Magnetic Fields of Industrial Frequency on Rat Liver Lipid Composition". Some of the interpretations are more fanciful than those allowed by refereed journals in the West. In many articles important experimental details, as well as the full address of the authors, are missing. So what you read, with difficulty, is all you get.

Soviet Progress in Biochemistry is not going to let Western biochemists know what Russian biochemists can contribute to our science. As progress in Western biochemistry is the direct result of sharing of information, reagents and trade, so perhaps glasnost and perestroika might result in some reversal of provincialism. Then we can relegate reading Progress in Soviet Biochemistry (in English) and Western Trends in Biochemistry (in Russian, if it exists) to the appropriate bureaucrats in Moscow, Washington and London, and get on with some real progress in Soviet biochemistry.

F.J. Bollum is a Professor in the Department of Biochemistry, Uniformed Services University of the Health Sciences, 4301 Jones Bridge Road, Bethesda, Maryland 20814, USA.

\section{It's only natural}

\section{Leslie Crombie}

Phytotherapy Research. Editor-in-chief Fred J. Evans. Heyden. 6/yr. $£ 118, \$ 190$.

MANKIND has been indulging in phytotherapy research for a long, long time, and the products of this research - traditional remedies - can be brews of a dozen or so useless plants along with one which contains an active and effective compound. Sorting out what, if anything, is effective in the remedy can be slow and difficult, but the occasional emergence of a compound with impressive pharmacological activity makes it all worthwhile.

In the editor's words, Phytotherapy Research is intended to fill a "need for a specialized journal to bring together the wealth of biological, therapeutic and analytical information on pure natural products, plant extracts and their pharmaceutical formulations". The journal publishes original research papers alongside rapid communications, reviews and book reviews. At the end of each issue there are two sections, a patent alert and a current literature bibliography. Given the widely scattered natural products literature relevant to this area, these sections are of great value for keeping up to date.

The journal will particularly appeal to pharmaceutical scientists. Some original papers report the screening of crude plant extracts, containing many chemical species, using a particular screen against certain disease conditions, thereby pro- viding leads for later phytochemical investigation and for the isolation and structural analysis of active chemical components. Others examine the biological activities of pure chemical compounds isolated from plants, sometimes chemically modified further. The chemical interest, however, apart from illustrative formulae, is rather limited in the four issues I looked at.

The journal seems reasonably priced and the general presentation of articles is modern and attractive. Publication appears to be quite rapid, inasmuch as this can be judged reliably for a new journal. There are of course other unknown quantities. A range of good manuscripts can usually be organized for the first few issues, but only time will tell whether the quality and flow can be sustained and developed. The level and extent of refereeing is not made clear, although this is of great importance for a new journal trying to establish a good reputation.

Nonetheless, Phytotherapy Research undoubtedly fills a need. It will be especially attractive as a forum for scientists from less-developed countries where phytotherapy is widely practised. Collaborations between scientists in developed and less-developed areas of the world give rise to especially valuable studies in this area of research, and some of the papers in the issues I examined bear that out.

Leslie Crombie is Sir Jesse Boot Professor in the Department of Organic Chemistry, University of Nottingham, Nottingham NG7 2RD, UK, and immediate Past Chairman of the Phytochemical Society of Europe. 